\title{
Developing the Athletic Training Clinical Scholar
}

\author{
Lindsey E Eberman \\ Indiana State University, Terre Haute, IN
}

Key Phrases

Practice-based research, practice-led research, quality improvement

\section{Correspondence}

Dr. Lindsey Eberman, Indiana State University, 567 N. 5th

Street, Terre Haute, IN 47809.

E-mail: Lindsey.Eberman@indstate.edu

Twitter: @isuathltraining

\section{Full Citation}

Eberman LE. Developing the Athletic Training Clinical

Scholar. Clin Pract Athl Train. 2019;2(1): 1-3.

https://doi.org/10.31622/2019/0001.1.

Submitted: February 3, 2019 Accepted: February 19, 2019

\section{EDITORIAL}

The 2020 Standards for Accreditation of Professional Athletic Training Programs ${ }^{1}$ requires that athletic training programs prepare their graduates to integrate the core competencies into their clinical practice. The core competencies derived from the former Institute of Medicine, ${ }^{2}$ and now National Academy of Medicine healthcare competencies, include patientcentered care, interprofessional and collaborative practice, evidence-based practice, quality improvement, health care informatics, and professionalism. Throughout these core competencies graduates are expected to use evidence to inform practice (Standard 62), use systems of quality improvement (Standard 63), and use data to drive informed decisions (Standard 64). ${ }^{1}$ In the development of a patient care plan, graduates will need to be able to assess the patient's status on an ongoing basis by collecting and analyzing patient-reported and clinician-rated outcomes (Standard 69). ${ }^{1}$ All of these tactics contribute to scholarly clinical practice by informing decisions locally, but could also inform the practice of others, thus having a global affect through dissemination as practicebased research.

As programs strategize how to teach these tactics to students, administrators are also likely deliberating how to support core faculty and planning for ongoing training of preceptors to demonstrate contemporary expertise. Programs should consider this an opportunity to meet all of the Standard expectations using the aforementioned scholarly clinical practice tactics and practice-based research. However, to effectively engage students, preceptors, and core faculty, program administrators must cultivate buy-in and expose everyone to different types of practice-based research. Specifically, a shift from faculty-led student scholarship to preceptorfacilitated clinical scholarship will need to occur. But this shift can't perpetuate conflicts between "the classroom" and "the athletic training facility" where these often assumptions between stakeholders in both environments that scholarly practices is not occurring. As these expectations shift, programs need to provide resources and help preceptors recognize that implementing these tactics will not only improve their clinical practice, but will also have the added benefits of enhancing clinical teaching and creating opportunities for collaboration with faculty to disseminate practice-based research.

Practice-based research involves clinicians answering relevant healthcare questions that matter to them and their patients, and translating research findings into practice. This can be achieved through a variety of scholarly activities. Examples of scholarship that require critical appraisal of the literature include evidence to practice reviews and validation case reports. In an evidence to practice review, a clinical scholar develops a question, hopefully about a problem they are trying to solve in their own practice, reviews the available literature, identifies a systematic review or meta-analysis, and helps to interpret and summarize this Level 1 evidence for others. To apply this evidence to practice, the clinical scholar can take the systematic review or meta-analysis and apply the research recommendations with one patient, or a series of 
patients, to determine if the best-evidence recommendations actually work in clinical practice. These are validation case reports and help us understand whether controlled studies with homogenous participant populations can translate in the real world. Athletic training students and preceptors can partner in development of evidence to practice reviews and validation case studies, especially as a scholarly activity early in an academic program where critical appraisal of the literature is the primary learning outcome.

Quality improvement is a systematic approach to analyzing clinical practice and improving performance. One of the first mechanisms of quality improvement is self-reflection, such as accurately viewing our own practice, and is achievable through tasks such as chart reviews. Reflection should be a regular habit in clinical practice, and can move from self-reflection to seeking feedback from others such as colleagues or a directing physician. The act of chart reviewing can also serve as a mechanism to explore shared characteristics of patient histories, how we use selective tissue testing in developing a differential diagnosis, rates of diagnoses, or time to recovery after using specific interventions. Document and chart reviews serve as an appraisal of one's own practice and help clinical scholars appreciate the landscape of the healthcare clinic or facility. They help us define what is currently happening and are the first step in the plan-do-study-act cycle of quality improvement. These are also a form of point-of-care research, particularly those that synthesize several patient cases to determine trends in practice. From a preceptor perspective, this might serve as a preliminary mechanism to teach athletic training students about the expectations of medical documentation while also helping to inform clinical practice decisions in the future.

Once a clinical scholar has a good understanding of the current practice landscape, they can begin to explore what changes need to be made to enhance efficiency by comparing current processes with those detailed as best-practices in the evidence. Then the clinical scholar can apply and study the change; analyze the data, and determine if the change resulted in the expected outcome. Finally, based on the data, clinical scholars will adapt, adopt, or abandon the change. This process, the plan-do-study-act cycle, is a form of quality improvement and can be documented and disseminated. The key to any quality improvement project is to understand that it is a continuous process, not just a one-time activity. So engaging students in this process regularly and continuously will socialize them to the tactics and make them habitual in clinical practice.

To effectively assess a patient's progress, athletic trainers need to be collecting and analyzing patient-reported and clinician-rated outcomes. Clinical outcomes research is a mechanism for communicating how measurement tools have been used in practice. Because these papers can be written from a variety of perspectives, clinical scholars could evaluate a specific outcome measure, or compare multiple measures in several patients from the same population or experiencing the same injury or illness. The application of outcome measures with one single patient is a great first step toward clinical integration. This data gathering technique can help with individual patient progress and ensure patient safety. As clinical scholars evolve, they should consider applying outcome measures to larger populations to determine clinician effectiveness, specifically regarding the interventions we apply. Clinical practice effectiveness of physical medicine and rehabilitation procedures is largely unknown, especially regarding interventions applied to patients that are not college-aged, white, or male. Unfortunately, most best-practice recommendations have been developed from high quality evidence, but in very homogeneous and often uninjured populations, which limits some of the recommendations. Clinical outcomes research can help develop evidence to support our decision-making, and because it is a tactic that we should be doing with each and every patient in their personalized care plans, it should be part of daily duties. As athletic training students move from interdependence to independent clinical 
care, one mechanism for practice performance evaluation in clinical education is a clinical outcomes research report, where the student demonstrates clinical competence through patient outcomes to preceptors and program personnel.

Clinicians hoping to integrate evidence into their practice are looking for clinically relevant research, which is evidence that comes from within practice and informs decisions at a global level. The traditional thesis is no longer relevant to meet these needs, especially for developing a clinical scholar. Professional masters programs should strongly consider these practice-based research initiatives as they meet multiple Standards 1 and outcomes of the program. These activities should transcend the program through sequentially planned experiences over the course of the curriculum. A constructivist approach that has students making meaning from experience to experience, reinforces the previous learning and minimizes the stigma of research in clinical practice. This is how we change the culture of athletic training and embrace evidence in clinical practice!

\section{REFERENCES}

1. Commission on Accreditation of Athletic Training Education. 2020 Standards for Accreditation of Professional Athletic Training Programs. Available at:

https://caate.net/wpcontent/uploads/2018/09/2020Standards-for-Professional-Programscopyedited-clean.pdf. Accessed on January 26, 2019.

2. Institute of Medicine. Crossing the quality chasm: A new health care system for the $21 \mathrm{st}$ century, Washington, DC; 2001. 